\title{
Quasidistributed fiber sensor for precast concrete structures monitoring
}

\author{
L. Rodriguez-Cobo ${ }^{\text {a }}$ J.A. Polanco ${ }^{b}$, A. Quintela ${ }^{\text {a }}$, I. A. Carrascal ${ }^{\text {b }}$ and J.M. López-Higuera ${ }^{\text {a }}$ \\ ${ }^{a}$ Photonic Engineering Group \\ ${ }^{\mathrm{b}}$ Dept. of Science and Engineering of Materials \\ University of Cantabria Avda. Los Castros s/n - c.p. 39005 Santander, Spain \\ Tel: +34-942-200877; Fax: +34-942-2000877; e-mail: rcobol@unican.es
}

\begin{abstract}
The prestressing force is transferred to the concrete during the slack period of precast concrete structures fabrication. To contribute to a better understanding of the armor compliant behaviors during the mentioned process, a custom quasidistributed Bragg Grating optical fiber sensor system is designed, fabricated and embedded into a prestressed concrete prismatic beam. The experimental works, the results, their discussion and finally the obtained conclusions are presented in this paper.
\end{abstract}

Keywords: Fiber Bragg Grating sensor, precast concrete, SHM, quasidistributed sensor.

\section{INTRODUCTION}

In the construction of precast concrete structures the armor is prestressed prior to the concreting process. Once the concrete is hardened and it has gained the adequate strength, the armor is slacked. During the slacking period, the prestressing force initially applied to the armor is transferred by adhesion to the concrete. The armor compliant behavior is something that, up today, has not been properly studied due to the fact that low reliable and low precision techniques are commonly used. The adherent nature of a prestressed reinforcement is defined through the conventional anchor length (Ls) after the unstressing fabrication process. As illustrated in Figure 1, conventional anchoring length Ls, in short, is defined as the length of the coating required to ensure the transmission of the maximum prestressing force which occurs on the concrete by releasing the ends of the reinforcement, initially tensioned to $80 \%$ of its nominal breaking load.

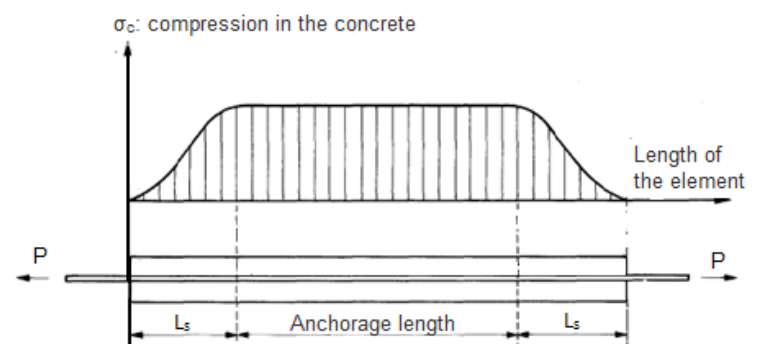

Figure 1. Expected compression force distribution after the unstressing fabrication process.

The standard UNE 7-436-82 "Bond test of steels wires for presstresed concrete" [1] indicates the methodology for determining this parameter and the validity criterion. The method proposed in the reference standard for determining the anchorage length consists in measuring the penetration length of each of the reinforcements of the concrete section after releasing the armor stress. This measurement was repeated after 1, 6, 24 and 168 hours. But these measurements are done actually with very traditional procedures: Several points are marked on the bean surface and their separation changes are followed (measured) during the slacking period of the beam fabrication process. This, today commonly used method, is unable to get real measurements of the inner part of the beam and, in addition, some measurements errors are commonly committed. 
A new fiber transducer based on Bragg grating technology and a new custom encapsulation is proposed to contribute to overcome the mentioned drawbacks. In this paper, the description of the new quasi-distributed optical fiber sensor system able to measure both the temperature and the strain/elongation along the axial axis (inside the beam), the experimental results their discussion and finally the obtained conclusions will be very briefly reported.

\section{OPTICAL FIBER TRANSDUCER}

The quasi-distributed transducer is based on Fiber Bragg Gratings (FBG) optical structure [2] what is being widely used in structural health monitoring [3]. As it is well known, the reflected wavelengths are centered around the Bragg wavelength $\left(\lambda_{B r a g g}=2 n_{e f f} \Lambda\right)$ and by measuring the central wavelength variations, the strain and/or the temperature of the fiber structure can be determined [4].

As illustrated in Figure. 2, the quasi-distributed transducer is integrated by FBS's properly placed inside a custom PMMA holder. To measure axial elongations, more than $20 \mathrm{FBG}$ 's are glued (using a cyanoacrylate) to the holding piece at fixed distances. Some FBG' are placed loosely (elapsed) to follow the inner beam temperature evolution and to be able to measure the real deformation. By combining the response of all the embedded FBGs along the beam, the deformation trend was obtained.

The PMMA holder is mechanized on piece of a constant thickness of $3 \mathrm{~mm}$ with a shape which favors its integration into the concrete. Two fixing areas surround each sensing point to force a better joint with the concrete, causing a homogeneous deformation on the central part of each sensing point. The FBG is glued to the bottom surface of a central groove made in the holding piece. This groove is also used to guide the optical fibers along the transducer, protecting it from possible hits or cracks during the installation. The central groove is sealed with thermofusible adhesive. The four optical input output fibers were protected with a plastic tube to reach the out edge of the beam.

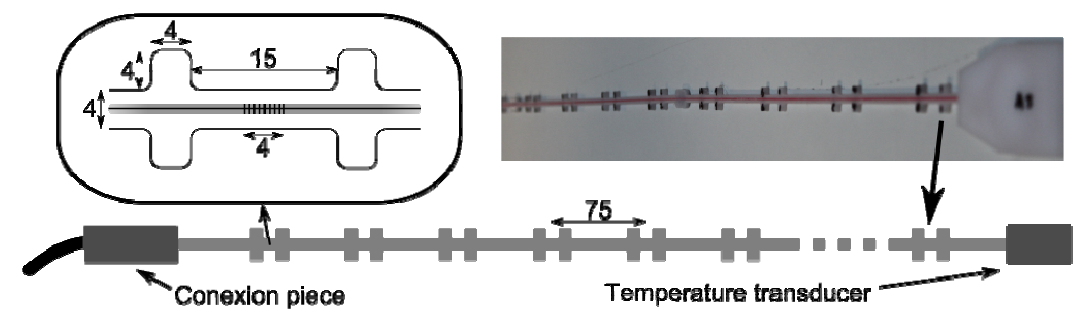

Figure 2. Transducer details. Dimensions are in millimeters.

\section{TRANSDUCER INTEGRATION AND BEAM FABRICATION}

As shown in Figure.3, the proposed transducer is installed in the axial central position of a prestressed prismatic concrete beam of rectangular section $\left(0.2\right.$ by $\left.0.15 \mathrm{~m}^{2}\right)$ of 3.55 meters of length. It is built using a concrete with a minimum value of resistance of $65 \mathrm{MPa}$ at 28 days. During the concreting process, three cylindrical test control samples were prepared $(\mathrm{h}=300 \mathrm{~mm}$ and $\varnothing=150 \mathrm{~mm})$ for determining the concrete compressive strength. On each of the reinforcement a strain gage will be placed in order to verify that no losses take place during the clamping operation.

The tensioning process of the reinforcements is performed by steps (at least three); recording the force and deformation of the reinforcement wires (the deformation is measured by strain gauge strips). These values are compared with those of the typical stress-strain diagram of the steel tested, with the purpose of avoiding any error in the assessment of the initial clamping force. After reaching the test force, the corresponding value obtained from the strain gauge, $\varepsilon_{a, i}$ is recorded. If during the process the force decreases, the hydraulic jack will be applied again until reaching the original value.The stretching is performed 24 hours before the concrete placing.

In order to get the adhesion properties of the prestressing reinforcement, the transducer is placed near one edge of the concrete beam to analyze its response evolution. It is fixed in the middle of the two steel reinforcement wires with nylon wires prior to concreting. The first sensing point is placed at $335 \mathrm{~mm}$ from the edge of the beam and the last a $1460 \mathrm{~mm}$. The distance between two consecutive sensing points is $75 \mathrm{~mm}$. 

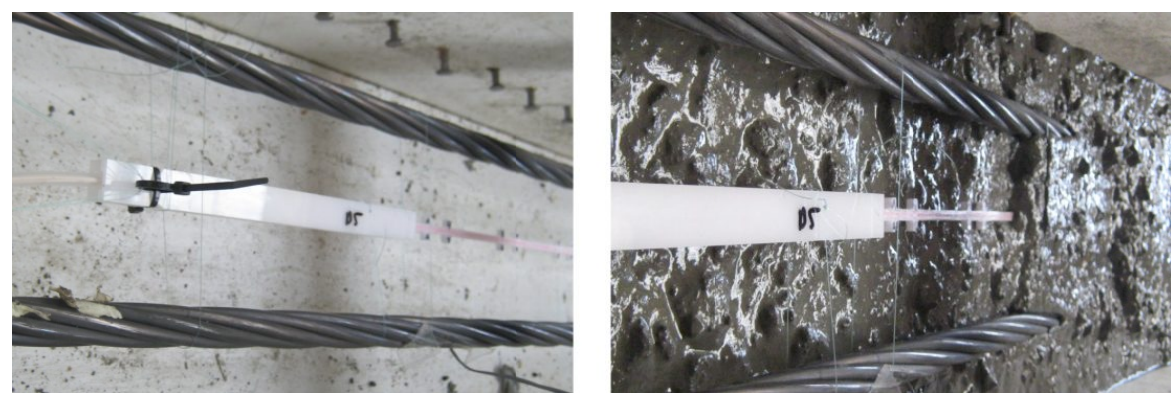

Figure 3. Installation details (left) and photo (right) obtained during the beam concreting process

After 24 hours from the tensioning process, the concrete pouring is made. After 5 days, when it was certain that the concrete had acquired an adequate strength, the stored samples were broken in order to confirm that the resistance exceeded the required value. The mean resistance of the three specimens reached $38.5 \mathrm{MPa}$, which is higher than the 24.5 MPa set by the standard as minimum test requirement.

Once the check is done, the releasing process of the reinforcement wires is initiated. The first operation consists in carefully removing the formwork and then placing two comparators with their corresponding supplement at each side of the beam, recording the initial values of penetration.

\section{RESULTS AND DISCUSSION}

The whole process has been monitored since the pouring process. The stress on the reinforcement wires is maintained during the concrete curing process. Once the beam is cured, a release stage of the reinforcement wires is followed and the deformation evolution of the beam is measured during 220 hours. The first interesting data coming from the installed transducer is the evolution of the temperature during the curing process of the beam. As shown in the Figure 4, from the $20^{\text {th }}$ hour the temperature establishes with a final rise of about $13^{\circ} \mathrm{C}$.

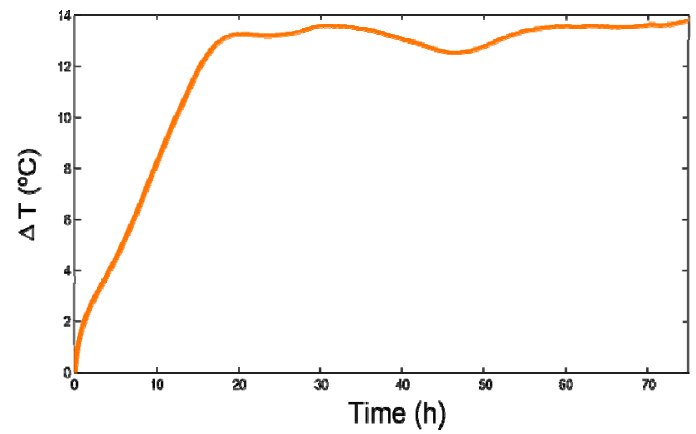

Figure 4. Temperature evolution during the concrete curing process

Once the beam is cured, the stress from the reinforcement wires is removed. For this process each wire is stressed to release the fixing means and after the load is reduced gradually. The process is repeated for each of both wires. The main deformation is produced in the first instants of the releasing process but the concrete continue comprising as the time passes. In the Figure 5.a is shown the evolution of compression for four points. In order to check the deformation evolution of the concreted beam, some checkpoints are taken at 1, 6, 24,168 and 220 hours from the releasing stage. This evolution is shown on the Figure 5.b. On this figure is also shown the curve which fits the deformation status at $\mathrm{t}=220$ hours after the releasing stage. The value of anchorage length is determined to be $1085 \mathrm{~mm}$, based on the estimate of the point of intersection of the two fit lines.

The anchor length will be determined according to the analyzed standard by calculating the average (Dm) of the penetrations in all reinforcements after seven days, and considering the anchoring conventional length equal to: 


$$
L_{s}=3,5 \frac{E_{s} \cdot A_{p n}}{f_{p m, G}} \delta_{m}
$$

Where $E_{s}$ is the modulus of elasticity of the active reinforcement $\left(E_{s}=200 \mathrm{GPa}\right) ; A_{p m}$ is the area of the active reinforcement $\left(A_{p n}=140 \mathrm{~mm}^{2}\right) ; f_{p m, G}$ is the guaranteed breaking load of active reinforcement $\left(f_{p m, G}=A_{p n} \cdot f_{\max , k}=140\right.$ $\left.\mathrm{mm}^{2} \cdot 1860 \mathrm{MPa}\right) ; \delta_{\mathrm{m}}$ is the average penetration of all reinforcements for three samples after 7 days. According to the standard, the anchor length of the passive side was $\mathrm{L}_{\mathrm{s}, \text { passive }}=1095 \mathrm{~mm}$ and the average anchor length obtained was $\mathrm{L}_{\mathrm{s}, \mathrm{m}}$ $=1054 \mathrm{~mm}$.
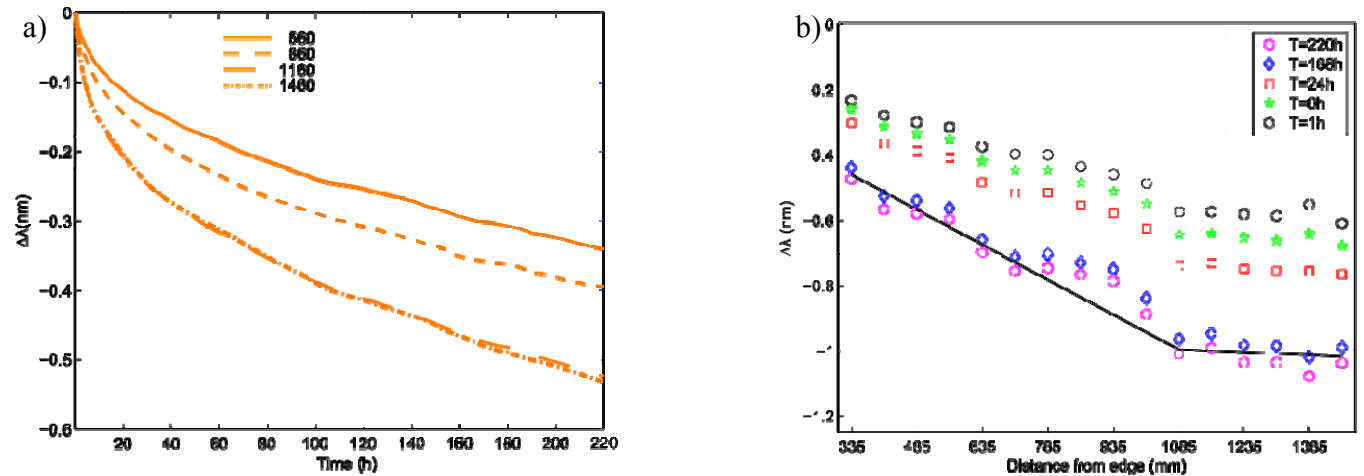

Figure 5. . a) Evolution of compression for four points ( 560, 860, 1160 and $1460 \mathrm{~mm}$ from the beam edge) b) Deformation of the 16 of the sensing points at different times: $1,6,24,168$ and 220 hours after releasing.

The results obtained by the transducer are the same that those calculated with the formulation proposed by the reference standard. The difference between the obtained values in the passive side and at the fiber optic transducer location is 10 $\mathrm{mm}$, resulting less than $1 \%$, with a $10 \%$ tolerance level in the standard when using an alternative method for determining the anchorage length

\section{CONCLUSIONS.}

Up today the anchoring length determination of an active reinforcement given by the UNE 7-436-82 standard is based on the registration of the penetration depth of the concrete's reinforcement using traditional (with low precision) measurement techniques. The feasibility of use fiber optic sensing technology has been demonstrated in this work. A quasi-distributed custom transducer based on FBG's technology has been proposed and fully developed. Placed and embedded in the axial core of the concrete beam, the proposed quasi-distributed fiber sensor enable a high precision monitoring of the elongation and temperature in a wide set of axial significant areas into the beam core-axis.

After the seven days proposed by the standard (UNE 7-436-82) for measuring the penetration, it is found that the deformations are not stable and the beam shortening process continues, what is in contradiction with the standard topic that is in the currents in study. This OFS technology will be proposed to be admitted by the standard for this purpose.

\section{ACKNOWLEDGEMENTS}

This work has been supported by the project TEC2010-20224-C02-02 and grant AP2009-1403. Roberto Perez Sierra is also thanked for his collaboration on the transducer fabrication.

\section{REFERENCES}

[1] AENOR. UNE 7-436-82 "Bond test of steels wires for prestressed concrete". 1982

[2] Kashyap, Raman 'Fiber Bragg Gratings', Academic Press, ISBN: 0-12-400560-8, 1999.

[3] Lopez-Higuera, J.M., Rodriguez Cobo, L., Quintela Incera, A., Cobo, A., "Fiber Optic Sensors in Structural Health Monitoring", Journal of Lightwave Technology, Vol. 29, Issue 4, Pages 587-608, 2011.

[4] J. M. Lopez-Higuera. Handbook of optical fibre sensing technology, Wiley \& Sons (2002). 